\title{
Fracture Criterion for the Tensile Test of 7075 Aluminum Alloy
}

\author{
D. C. Chen ${ }^{1}$ and C. S. You
}

Department of Industrial Education and Technology, National Changhua University of Education, Changhua, Taiwan

${ }^{1}$ dcchen@cc.ncue.edu.tw

True stress-strain values were obtained from the tensile tests of 7075 aluminum alloy and finite element analysis. The results revealed that different ductile fracture criteria resulted in different accuracy of analysis. Moreover, factors affecting ductile fracture, such as effective stress, effective strain, and damage were analyzed. The maximum damage level occurred in the center, with its value reaching 0.454. The break happened in $12.9 \mathrm{~s}$ in the P1 point. In the P3 point, fracture was not revealed. It can be seen that the maximum damage was observed over the fracture area in the P1 point. Finite element analysis as applied to the 7075 aluminum alloy fracture criteria can be used for forging, drawing, and stamping of the materials.

Keywords: 7075 aluminum alloy, damage value, tensile test.

Introduction. 7075 aluminum alloy exhibits a high strength-to-weight ratio and is widely used in aircraft and aerospace components, such as wings and fuselage. Due to ductility of this material, it is important to use the appropriate ductile fracture criterion in calculations of components produced from it.

In numerous studies, tensile tests have been performed to determine the ductile fracture of materials. Weng and Sun discussed different fracture criteria for ductile materials, using the finite element analysis [1]. Komori analyzed various ductile fracture criteria for simulating shear by separation of the finite element nodes [2]. Yang et al. performed damage analysis and studied fracture criteria for various materials [3]. Hambli and Reszka provided the fracture criteria identification using an inverse technigue method and blanking experiment [4]. The calculation results obtained by six fracture criteria had the same correlation with the experimental data, while the best fit was provided by the normalized Cockcroft-Latham fracture criterion. Hoa et al. simulated the metal formation process in the cold forging using a finite element fracture zone [5]. Narayanasamy et al. studied the extrusion-forging process at room temperature [6]. This process was characterized as simple and economical in mass production, since metallic materials processed through plastic deformation change their internal structure, thus improving their mechanical properties. Yang et al. studied the surface stretch forming process in 2060-T8 aluminumlithium alloy, where formation of an orange peel structure was observed [7]. Zhu et al. used a finite element model to simulate the fluid-solid interaction in porous materials [8]. Jia et al. investigated microstructure and mechanical properties of 2219 aluminum alloy after solid solution treatment [9].

In this study, tensile tests of 7075 aluminum alloy are conducted to determine the true stress-strain state and to elaborate the appropriate ductile fracture criterion, according to the finite element simulation analysis..

Experimental Method and Finite Element Analysis. In order to derive the flow stress of a plastic material obtained from the stress-strain relationship, the finite element method within the elastoplastic approach can be used. After obtaining an engineering strain diagram by performing the tensile test, the following formulas can be used to construct the true stress-strain diagram:

$$
\sigma=S(1+\delta),
$$




$$
\varepsilon=\ln (1+\delta),
$$

where $\sigma$ is the true stress, $S$ is the engineering stress value, $\delta$ is the engineering strain value, and $\varepsilon$ is the true strain.

While brittle fracture of metallic materials occurs mainly at the grain boundaries, ductile failure is frequently related to crystalline grain boundary sliding caused by shear damage. Ductile failure can also be treated as plastic deformation of the nonmetallic material present in the inclusions with further precipitatation of fine particles, formation of voids, their growth, and the ultimate fracture. Ductile failure is controlled by the processing parameters such as the rate of deformation and molding conditions, lubrication, and friction. In addition, any alterations to the material chemical composition affect its microstructure and the uniformity of its surface.

Rigid-plastic finite element DEFORM ${ }^{\mathrm{TM}} 3 \mathrm{D}$ software is widely used for simulation of forging, extrusion, pulling, rolling, stamping, upsetting, and other forming processes of metals. This software, structure of which can be subdivided into four modules, such as: pretreatment, simulation engine, post-processor, and multifunction, provides simulation of the plastic flow stress. The flow stress equation has the following form:

$$
\bar{\sigma}=\bar{\sigma}(\bar{\varepsilon}, \dot{\bar{\varepsilon}}, T)
$$

where $T$ is temperature, $\bar{\varepsilon}$ is strain, and $\dot{\bar{\varepsilon}}$ is strain rate.

Because of the variable friction forces acting at the contact surfaces, the uniform friction model is based on the following dependence between the tangential stress and metal-tool slip velocity:

$$
\tau_{f}=-m k \frac{2}{\pi} \arctan \left(\frac{\left|v_{p}\right|}{a_{p}}\right) \frac{v_{p}}{\left|v_{p}\right|},
$$

where $\tau_{f}$ is shear stress of friction, $m$ is the friction factor, $k$ is the local flow stress in shear, $v_{p}$ is the slip velocity vector, and $a_{p}$ is the coefficient, which is less than the slip velocity by several orders of magnitude.

The maximum damage value obtained and the value corresponding to the critical damage of the material (critical damage value) can be used to predict the ductile behavior of arrow-shaped cracks originating from the molding damage [10]. The maximum value of the damage and fracture condition are attained, when the material is formed under the maximum tensile stress conditions, as is shown by Eq. (5). Chen et al. [11] introduced the normalized Cockcroft-Latham criterion, by normalizing Eq. (5) and reducing it to the dimensionless form of Eq. (6):

$$
\begin{aligned}
& \int_{0}^{\bar{\varepsilon}_{f}} \sigma_{\max } d \bar{\varepsilon}=C, \\
& \int_{0}^{\bar{\varepsilon}_{f}} \frac{\sigma_{\max }}{\bar{\sigma}} d \bar{\varepsilon}=C .
\end{aligned}
$$

Results and Discussion. To determine the plastic flow stress of 7075 aluminum alloy, tensile tests were performed using standard cylindrical specimens of the total length of 220 $\mathrm{mm}$ with the working part having diameter $D=14 \mathrm{~mm}$ and length $L=60 \mathrm{~mm}$ (Fig. 1). The strain gauge was glued to the working portion surface, as shown in Fig. 2, and the specimens were subjected to tensile loading via a universal testing machine. 


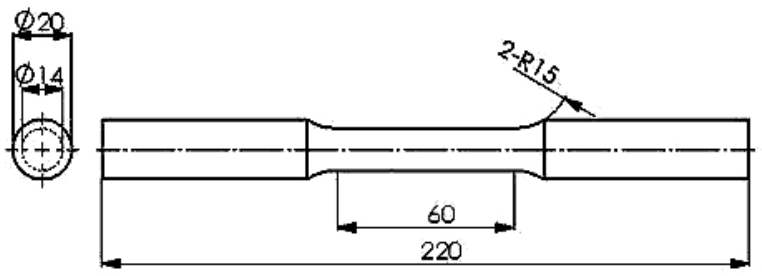

Fig. 1. Tensile test bar size.

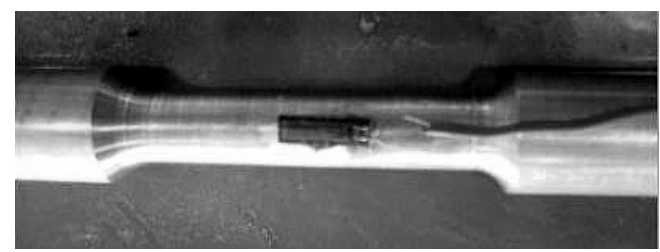

Fig. 2. The strain gauge position.

Figure 3a-c depicts the tensile test simulation before loading, during loading, and after fracture, with the tensile forces being applied at the specimen edges. It can be seen that the initial strains are evenly distributed along the working portion length, while the material plastic flow occurs when the fracture stress value is attained. Figure $3 \mathrm{~d}$ depicts the enlarged view of the final fracture zone.

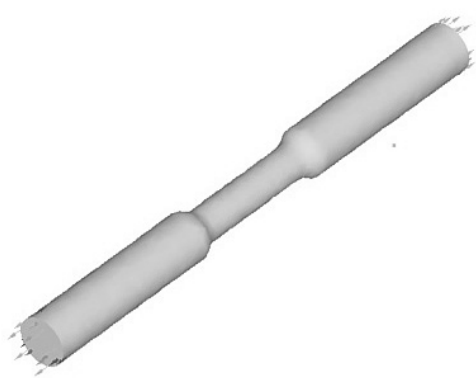

$\mathrm{a}$

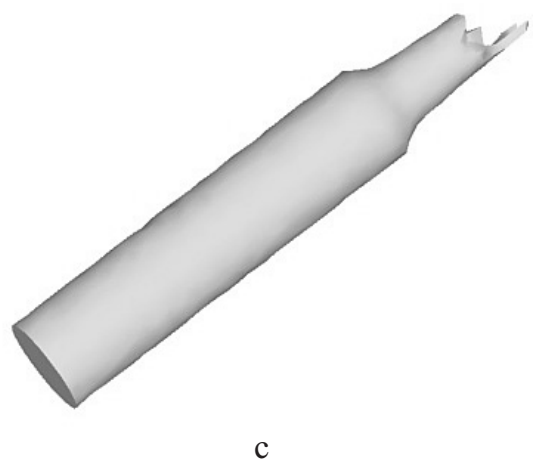

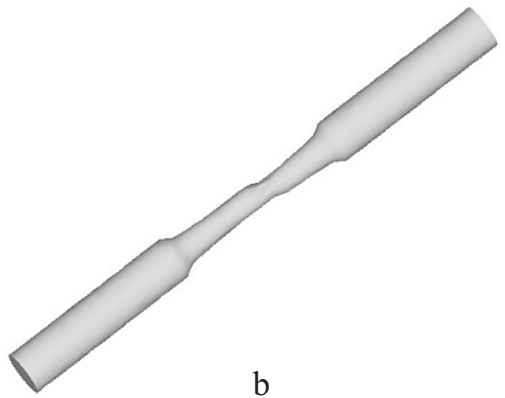

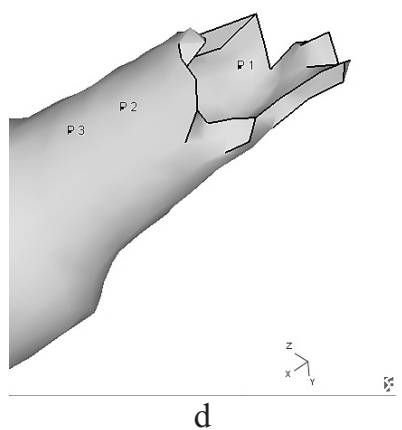

Fig. 3. Schematic diagram of the tensile test simulation: (a) before tensile; (b) during tensile; (c) after tensile fracture; (d) after tensile fracture in analysis point.

Figure 4a-c displays the effective strain diagram for the 7075 aluminum alloy, revealing that the maximum effective strain occurs in the midddle point, which corresponds to the maximum strain value and, thus, is the most susceptable to fracture. Figure $4 \mathrm{~d}-\mathrm{f}$ 
depicts the effective stress diagrams, where the points with the maximum stress and the maximum effective strain are assumed to coincide, thus producing stress concentration points, from which cracks may originate. Moreover, it can be seen that the point with the maximum effective stress value is located if the fracture area of the tear-shaped when the effective stress reaches $91 \mathrm{MPa}$.

Figure 5a depicts the effective strains after tensile fracture in points $P 1, P 2$, and $P 3$ location of which is shown in Fig. 3d: fracture occurred at the time of $12.9 \mathrm{~s}$ in point $P 1$, while no fracture condition was reached in point $P 3$. Figure $5 \mathrm{~b}$ shows the effective stress diagram for the above points. Here the final rupture time was $12.9 \mathrm{~s}$, when the stress made a sharp drop and the maximum effective stress was generated in the fracture zone.

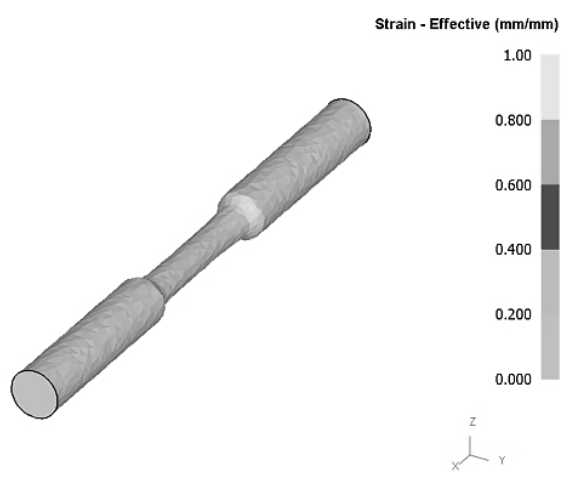

$\mathrm{a}$

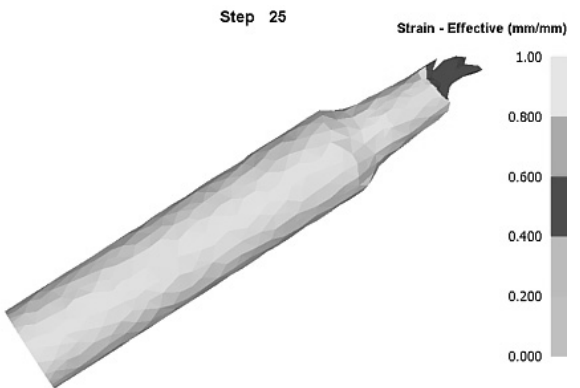

$\mathrm{c}$

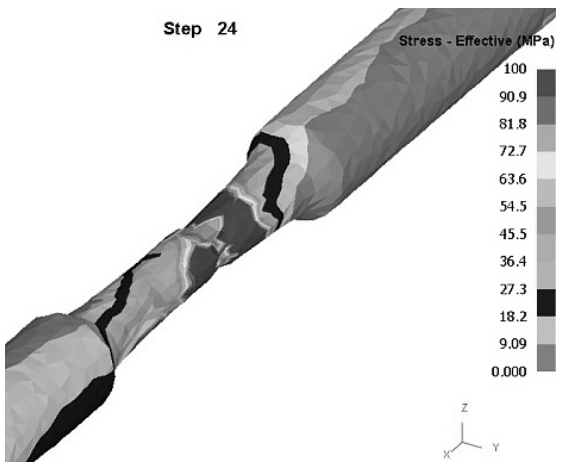

e

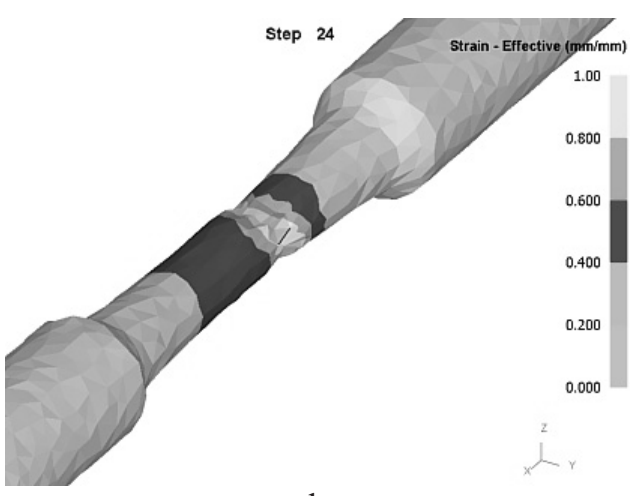

b

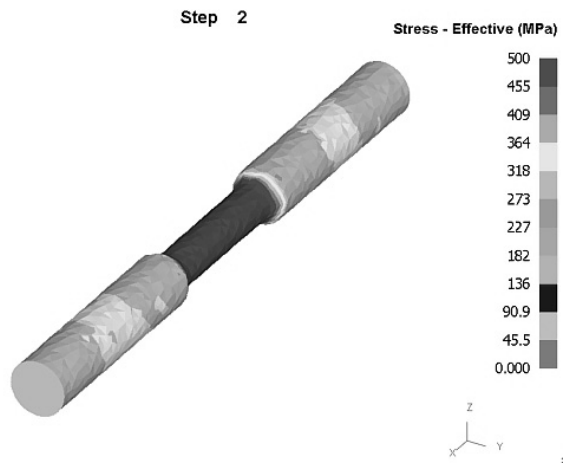

$\mathrm{d}$

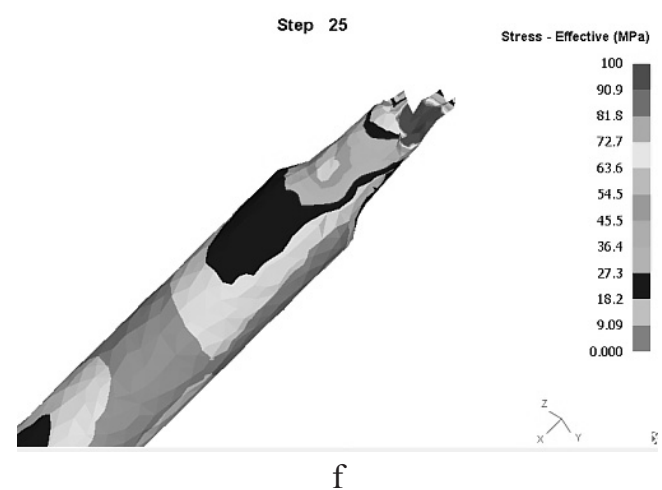

Fig. 4. 7075 aluminum alloy effective strain $(a-c)$ and stress $(d-f)$ diagrams: (a, d) initial tensile effective strain and stress diagrams; $(b, e)$ effective strain and stress graphs before tensile fracture; $(c, f)$ effective strain and stress diagrams after the tensile fracture. 


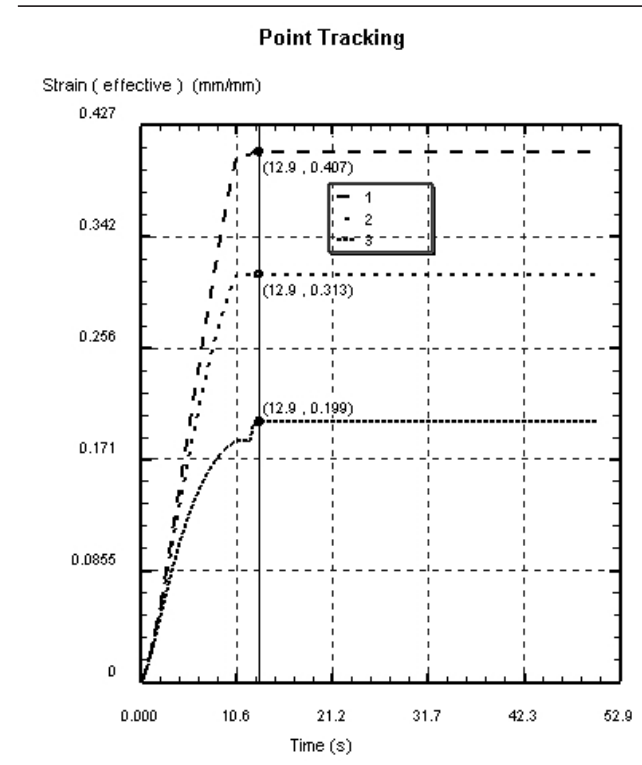

a
Point Tracking

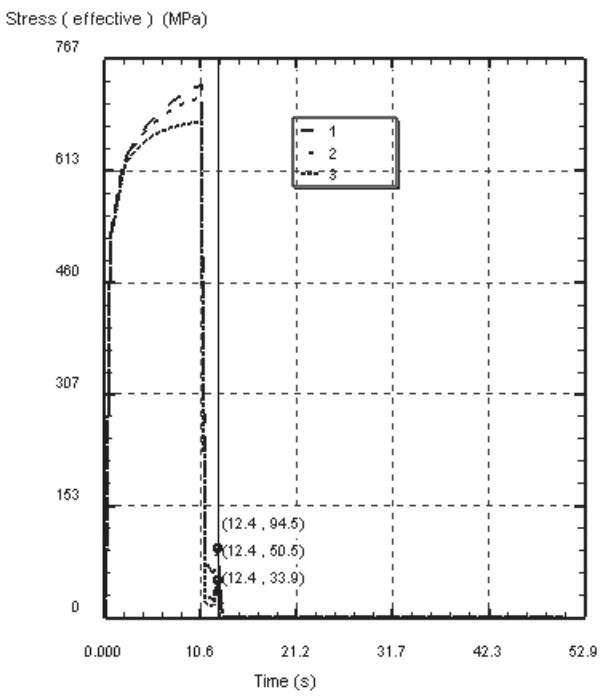

b

Fig. 5. 7075 aluminum alloy effective strain (a) and stress (b) diagrams in the tensile test.

Figure 6 depicts the damage evolution for the 7075 aluminum alloy for the case, where the damage value predicted by the normalized Cockcroft-Latham criterion was equal to $C=0.454$. Thus, damage values over 0.454 would produce the grid separation (fracture), resulting in the speciment fracture in the initial point. It can be seen that the maximum damage value was attained in the central point, when the damage value reached 0.454 [4].

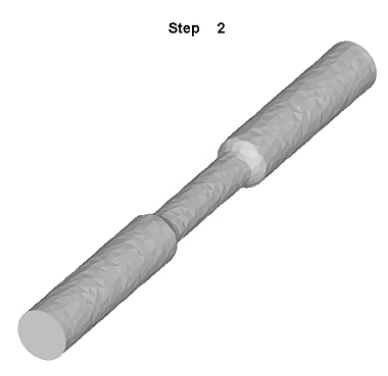

a

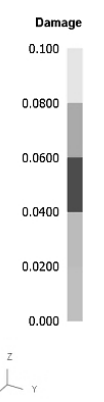

r

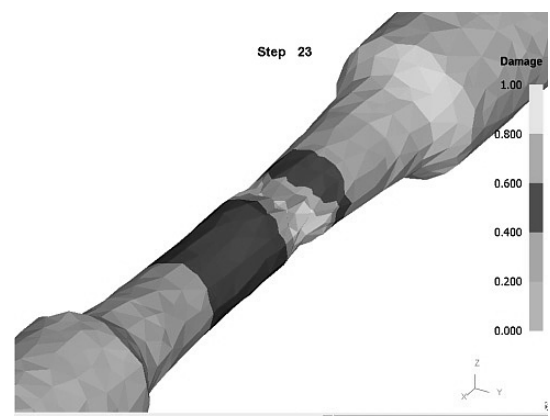

b

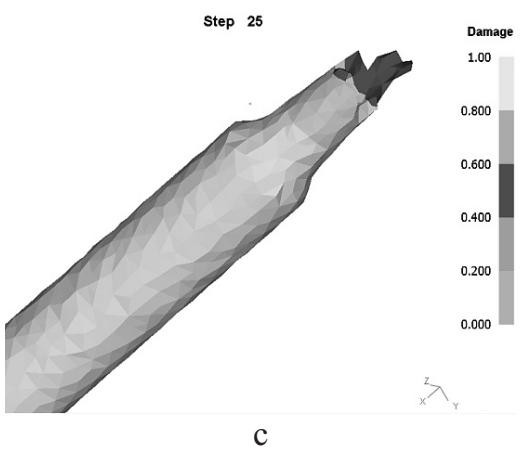

Fig. 6. Damage diagram of 7075 aluminum alloy: (a) preliminary damage tensile values; (b) damage value before tensile fracture; (c) damage value after tensile fracture. 
Point Tracking

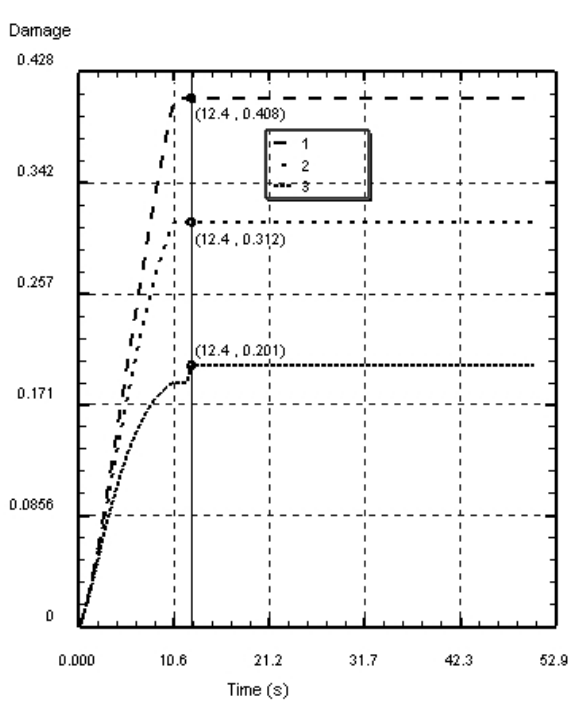

Fig. 7. 7075 aluminum alloy damage value after tensile fracture in analysis diagram in analysis point.

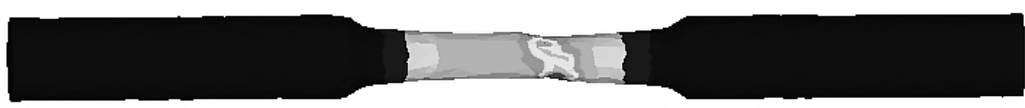

a

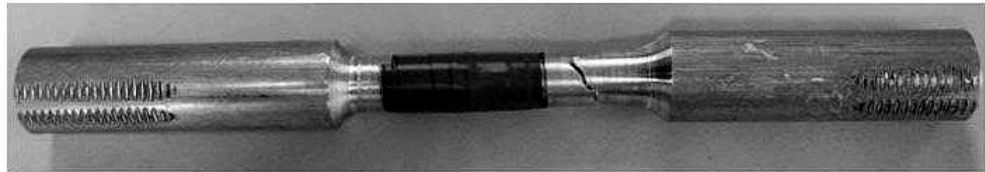

$\mathrm{b}$

Fig. 8. 7075 aluminum alloy fracture situation: (a) fractured simulation; (b) fracture experiment.

Figure 7 illustrates the damage evolution in the analyzed points: the maximum damage value is attained in the fracture area in point $P 1$. Figure 8 depicts the experimental and simulated fracture patterns, which show a good correlation.

Conclusions. True stress-strain values were obtained from the tensile tests of 7075 aluminum and finite element analysis. Factors affecting ductile fracture, such as effective stress, effective strain, and damage were analyzed. The maximum damage level occurred in the center, with its value reaching 0.454 . The break happened in $12.9 \mathrm{~s}$ in the $P 1$ point. In the $P 3$ point, fracture was not revealed. It can be seen that the maximum damage was observed over the fracture area in the $P 1$ point. Finite element analysis as applied to the 7075 aluminum alloy fracture criteria can be used for forging, drawing, and stamping of the materials.

1. T. L. Weng and C. T. Sun, "A study of fracture criteria for ductile materials," Eng. Fail. Anal., 7, 101-125 (2000).

2. K. Komori, "Ductile fracture criteria for simulating shear by node separation method," Theor. Appl. Fract. Mech., 43, 101-114 (2005).

3. X. Yang, C. Chen, Y. Hu, and C. Wang, "Damage analysis and fracture criteria for piezoelectric ceramics," Int. J. Non-Linear Mech., 40, 1204-1213 (2005). 
4. R. Hambli and M. Reszka, "Fracture criteria identification using an inverse technique method and blanking experiment," Int. J. Mech. Sci., 44, 1349-1361 (2002).

5. V. C. Hoa, D. W. Seo, and J. K. Lim, "Site of ductile fracture initiation in cold forging: a finite element model," Theor. Appl. Fract. Mech., 44, 58-69 (2005).

6. R. Narayanasamy, K. Baskaran, and D. Muralikrishna, "Some studies on stresses and strains of aluminium alloy during extrusion-forging at room temperature," Mater. Des., 29, 1623-1632 (2008).

7. L. Yang, L. H. Zhan, and W. Wu, "Analysis method of orange peel structure on aluminum-lithium alloy surface during stretch forming process," Mater. Res. Innov., 18, Issue S2, 5-11 (2014).

8. Q. J. Zhu, Y. F. He, and Y. Yin, "Finite element analysis of deformation mechanism for porous materials under fluid-solid interaction," Mater. Res. Innov., 18, Issue S2, 22-27 (2014).

9. S. F. Jia, L. H. Zhan, and J. Zhang, "Influence of solid solution treatment on microstructure and mechanical properties of 2219 aluminum alloy," Mater. Res. Innov., 18, Issue S2, 52-58 (2014).

10. V. T. Troshchenko and L. A. Khamaza, "Conditions for the transition from nonlocalized to localized damage in metals and alloys. Part 1. Crack sizes at fatigue limit," Strength Mater., 46, No. 3, 303-314 (2014).

11. C. C. Chen, S. I. Oh, and S. Kobayashi, "Ductile fracture in axisymmetric extrusion and drawing," J. Eng. Ind., 101, 36-44 (1979). 